\title{
Voluntary Blinks Stop Binocular Rivalry
}

\author{
Ahmad Yousef ${ }^{1}$ \\ ${ }^{1}$ School of Computational Science and Engineering, McMaster University, Hamilton, Ontario, Canada \\ *Correspondence: mohamas2@mcmaster.ca
}

\begin{abstract}
This article provides evidence that voluntary blinks are able to indefinitely stop binocular rivalry; namely after each blink, human subjects had reported seeing exclusively an unchangeable stimulus. The subjects, also after each blink, had noticed that the content lines of the visually dominant stimulus are getting much wider, and the stimulus itself is getting clearer; before it returns to its initial state. We therefore asked the subjects whether they can stop the rivalry indefinitely, through certain blinking procedure; and all of them had validated their ability.

\section{Introduction}

Binocular rivalry, a phenomenon of visual perception in which human visual awareness alternates between different visual stimuli presented to each eye, is an astonishing psychophysical approach to quantify consciousness. Noticeably, both kinds of attention, involuntary or voluntary, assist in increasing the visual consciousness dominance duration of the corresponding stimulus. In another word, attention controls the human visual awareness.

Strength (Levelt, 1965), saliency (Engle,
\end{abstract} 1956), spiral motion (Malek, 2012), higher spatial frequency (Fahle, 1982), duchenne expressions (Malek, 2018), are all having 'attractive' visually stimulating features, namely, they all trigger automatic attention; therefore they all dominate the visual awareness over 'nonattractive' ones. Voluntary attention to certain details to a certain

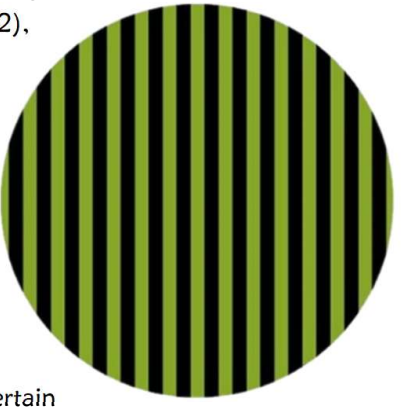
stimulus also elongates the awareness dominance duration of the stimulus (Lake, 1978).

All of the aforementioned experiments, however, were not able to cease the rivalry indefinitely, namely, they were able to increase the visual awareness dominance duration, but not to stop the rivalry itself. The first trial to stop the rivalry indefinitely was done by Olivia Carter and her colleagues, see reference 7; which was achieved by the meditation of Tibetan Buddhist monks. In this letter, however, we are offering a novel procedure that allows human subjects to indefinitely stop their binocular rivalry.

\section{Materials \& Methods}

Twelve human subjects, with average pupils, were recruited to participate in this study. Each circle in each stimulus subtends exactly 8.76 DVA, namely, 3.7 centimeters, on the screen, whereas the human subjects are 24 centimeters away from the screen, with 9.25 diopters base-out glasses, see reference 8 . Important to mention, we had used different stimuli than the one on the center of this page, namely, our tested stimuli are white and black. The colored ones are offered for the readers who don't have a professional binocular rivalry system; but they would like to have a closer experience of what we are reporting in this study. To experience binocular rivalry, just open this paper on your cell phone or tablet, approach your eye to the screen until the to circles (green and red) seem to merge into one circle. Once they are perfectly merged, binocular rivalry will start, namely, the green and the red circles will rival each other to access the visual awareness. At this moment, you can start to blink to 'informally' validate our current theory. Importantly, we had used those colored stimuli for nothing but to ease the procedure of that 'informal experiment'; which will nicely work, but it is not very professional though! Back to our preliminary experiment, we had four trails for each of our twelve human subjects; the first two trails' stimuli are built through horizontal lines for the right-side stimulus versus vertical lines for left-side stimulus; and eventually, the aforementioned trail will be counterbalanced. The next two trails' stimuli are built through tilted lines $\left(45^{\circ}\right)$ for the right-side stimulus versus tilted lines $\left(135^{\circ}\right)$ for the right-side; and eventually, the previous trail will be counterbalanced.

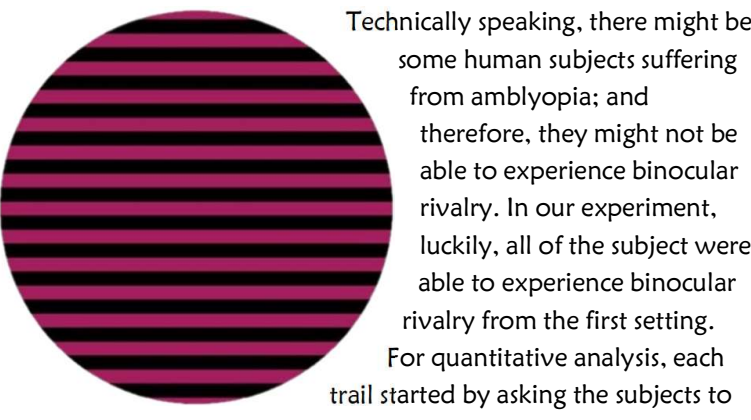
report every switch in a normal binocular rivalry setting for five minutes, whereas a specialized timer had been recording every switch, key press 1 for right-sided stimulus, and key press 2 for the left-sided stimulus. The second part of each trail is to request the subject to perform voluntary blinks with very special procedure. Preliminary speaking, the twelve human subjects had noticed that after each blink, there must be unchangeable stimulus reappears in the visual awareness, but not the other; the unchangeable stimulus, however, varies from subject to another. We had noticed that the bars are getting much wider, and the image is getting clearer of the visually dominant stimulus, before it returns to its initial state, and afterwards, the suppressed stimulus will start to invade the visual awareness. We therefore had utilized these observations into our design, by asking the human subjects to blink just before the dominate stimulus tries to return to its initial state; and to continue to blink whenever this scenario is willing to happen; a process to avoid the appearance of the rivalrous stimulus. Noticeably, the twelve subjects had observed that a perceptual switch must happen just after any 'voluntary' blink which brings the awareness of an unchangeable stimulus, namely, the 'same' stimulus, but not the other, must reappear 
after every 'voluntary' blink. The timing of the voluntary blinks must be considered as a very critical issue, otherwise, the indefinite stoppage will collapse. Need to emphasize, the human subjects have to blink just a few milliseconds after the dominate stimulus returns back to its initial state, otherwise, the suppressed stimulus will start to invade the awareness. While collecting the behavioral data, and to comply to Helsinki declaration, however, we requested from the subjects to stop blinking after five minutes of unstoppable blinking. All of the subject, expectedly, were able to stop the rivalry throughout the allocated five minutes.

Results and Discussion As shown in the central infographic, voluntary blinks were able to stop the rivalry for the entire second session of each trail, namely, five minutes of stoppage. To easily visualize the data, we applied the common logarithm for the dominance duration difference (average duration of dominate stimulus minus average duration of suppressed one) to show visible comparison between five minutes, and a few seconds. Noticeably, the dominance duration difference

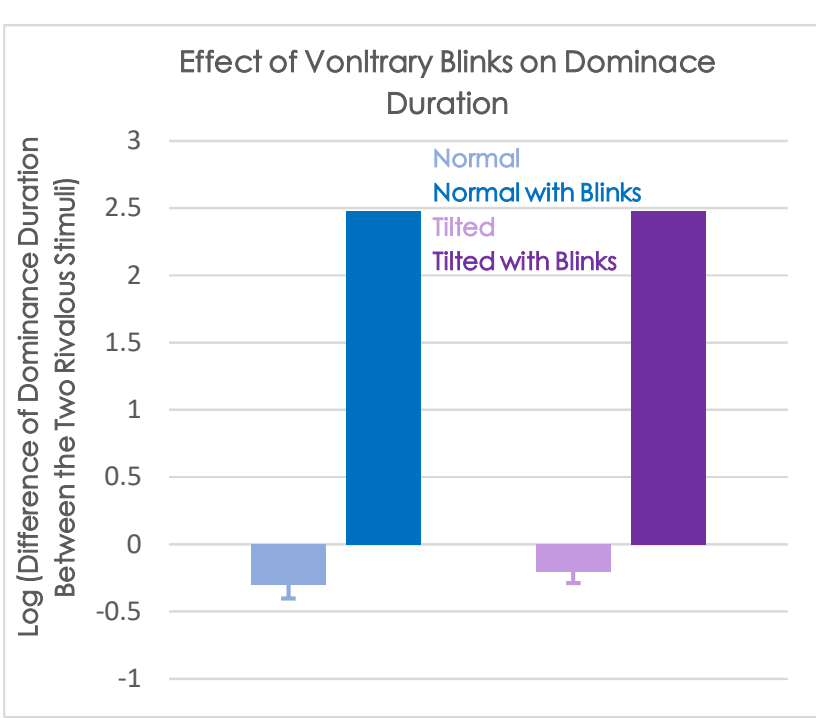

corresponding photoreceptors, because indefinite GABA machinery activation might be biological impossible. Finally, although (van Dam, et al. 2005) has concluded that eye movements, including spontaneous blinks, were not essential to perceptual switches, namely, they claimed that there is no causal correlation between the perceptual switch and blinks that occurred prior to switches; we however, based on our current observations, believe that there is a clear distinction between voluntary and involuntary blinks! Needless to mention, this research had confirmed that the pupil dilation could perceptually elongate the length (wider lines after each blink), namely, the dynamical visual angle should be considered. see the procedural steps, and reference 14. Essential Notification Stimuli and the BR setup can be found in reference 8 . Readers who don't have binocular rivalry setup are welcome to visit our lab; they can also make an initial confirmation using the stimuli provided in page 1 , but it must be opened from a cell or a tablet device, and the two circles must be merged into one circle to have the experience. Scholars with brain imaging and retinal

of the tilted stimuli is greater than horizontal/vertical ones. Voluntary blinks, the voluntary intermitted presentation, might result consistency with the fact that the dominance duration can be prolonged when the stimuli are presented intermittently (Leopold et al., 2002). The indefinite stoppage, however, might be a product of the astonishing voluntary attention achieved by the human subjects. Noticeably, immediately after each blink the pupil must be temporally dilated and then returns back to its normal state. That phasic dilation might be one of the causes of the current indefinite stoppage, given the fact that temporal phasic pupil dilation reflects perceptual selection $(W$. Einhäuser, etal. 2008). It is important to mention that GABA concentration has a positive correlation with the dominance duration of binocular rivalry stimuli (van Loon et al., 2013). Stimulation of $\mathrm{GABA}_{\mathrm{A}}$ receptors with Lorazepam shows a similar effect (van Loon et al., 2013); as if there is a positive correlation between voluntary blinks and the activation of GABA machinery. We have previously theorized that the inhibitory neurons had been sending information to the consciousness, and the consciousness has spectrum, starting from oblivion and ending with metacognition, see reference, 12 . We have to stop here for a moment, however, because based on van Loon et al., 2013 previous findings; indefinite stoppage against binocular rivalry must require indefinite 'local' activations of GABA machinery that exceed the human biological capabilities; and this might never happen. Our entanglement theory, therefore, might have received an additional supporting evidence, see reference 12 . Namely, we might conclude that the voluntary blinks affect the consciousness in a way that enables the awareness to continuously build entangled wave functions between the emitted photons of the dominate visual stimulus and its imaging facilities are welcome to cooperate. Enquiries should be sent to the author.

Transactional References

[1] Levelt (1965). Binocular brightness averaging and contour information. British Journal of Psychology.

[2] Engle (1956). The role of content in binocular resolution. The American Journal of Psychology.

[3] Malek, etal. (2012). Binocular rivalry of spiral and linear moving random dot patterns in human observers.

[4] Fahle (1982). Binocular rivalry: suppression depends on orientation and spatial frequency. Vision Research.

[5] Malek, (2018). Generalizing Duchenne to sad expressions with binocular rivalry and perception ratings. Emotion.

[6] Lack (1978). Selective attention and the control over binocular rivalry, Dissertation.

[7]O. Carter, etal. (2005). Meditation alters perceptual rivalry in Tibetan Buddhist monks. Current Biology.

[8]Stimuli and the BR system may be found here:

https://drive.google.com/drive/folders/13FoBh7E6KGxfp5ig-b833OvIAizQddzF [9] D. Leopold, etal. (2002). Stable perception of visually ambiguous patterns. Nature Neuroscience.

[10] W. Einhäuser, etal. (2008). Pupil dilation reflects perceptual selection and predicts subsequent stability in perceptual rivalry. Proceedings of the National Academy of Sciences.

[11] van Loon et al., (2013). GABA shapes the dynamics of bistable perception. Current Biology.

[12] Yousef, Ahmad. 2019. "Consciousness Might Be Localized in Extra Physical Dimensions." PsyArXiv. 2019. doi:10.31234/osf.io/angc8.

[13] Loes van Dam, and Raymond van Ee (2005). The role of (micro)saccades and blinks in perceptual bi-stability from slant rivalry. Vision Research.

[14] Yousef, Ahmad. 2019. "The Dynamical Visual Angle." PsyArXiv. doi:10.31234/osf.io/f2kr6. 